EPJ Web of Conferences 57, 02006 (2013)

DOI: $10.1051 /$ epjconf/20135702006

(C) Owned by the authors, published by EDP Sciences, 2013

\title{
Frequency metrology in quantum degenerate helium
}

\author{
Wim Vassen
}

\author{
LaserLaB VU University, Department of Physics and Astronomy, De Boelelaan 1081, \\ 1081 HV Amsterdam, The Netherlands
}

\begin{abstract}
We have measured the absolute frequency of the 1557-nm doubly forbidden transition between the two metastable states of helium, $2{ }^{3} \mathrm{~S}_{1}$ (lifetime $8000 \mathrm{~s}$ ) and $2{ }^{1} \mathrm{~S}_{0}$ (lifetime $20 \mathrm{~ms}$ ), with $1 \mathrm{kHz}$ precision. With an Einstein coefficient of $10^{-7} \mathrm{~s}^{-1}$ this is one of weakest optical transitions ever measured. The measurement was performed in a Bose-Einstein condensate of ${ }^{4} \mathrm{He}^{*}$ as well as in a Degenerate Fermi Gas of ${ }^{3} \mathrm{He}^{*}$, trapped in a crossed dipole trap. From the isotope shift we deduced the nuclear charge radius difference between the $\alpha$-particle and the helion. Our value differs by $4 \sigma$ with a very recent result obtained on the $2{ }^{3} \mathrm{~S} \rightarrow 2{ }^{3} \mathrm{P}$ transition.
\end{abstract}

\section{INTRODUCTION}

High resolution laser spectroscopy is a prominent tool to test the theory of atomic structure, in particular Quantum Electrodynamics (QED). Especially for the lightest atoms, helium and hydrogen, theory has reached a very high level and is tested with ever increasing accuracy since the advent of tunable lasers since the 1970's. Concentrating initially primarily on atomic hydrogen, progress in variational calculations since the 1990's has increased the accuracy with which helium energy levels can be calculated considerably. Part of this effort was motivated by the possibility to extract the fine structure constant from the splitting of the $1 \mathrm{~s} 2 \mathrm{p}{ }^{3} \mathrm{P}$ state. As QED theory provides the largest shift for lowlying $1 \mathrm{sns}$ states, a lot of effort is put in measuring the $1 \mathrm{~s}^{2}{ }^{1} \mathrm{~S}_{0}$ ground state and $1 \mathrm{~s} 2 \mathrm{~s}{ }^{1} \mathrm{~S}_{0}$ and $1 \mathrm{~s} 2 \mathrm{~s}{ }^{3} \mathrm{~S}_{1}$ metastable states (lifetimes $20 \mathrm{~ms}$ resp. $8 \mathrm{ks}$ ) energies of ${ }^{4} \mathrm{He}$. Apart from QED terms, tiny shifts, orders of magnitude smaller than the QED shifts, are caused by the finite size of the nucleus, also best probed in S-states. The present status of theory does not yet allow accurate extraction of nuclear charge radii from absolute level energies, however, in the isotope shift between ${ }^{3} \mathrm{He}$ and ${ }^{4} \mathrm{He}$ QED uncertainties are very small and mean square charge radius difference between the $\alpha$-particle and the helion $\left({ }^{3} \mathrm{He}\right.$ nucleus) can be determined with high accuracy.

We present here the observation of the $2^{3} \mathrm{~S} \rightarrow 2{ }^{1} \mathrm{~S}$ magnetic dipole transition between both metastable states. With a natural lifetime of $8 \mathrm{~Hz}$ (determined by two-photon decay of the $2{ }^{1} \mathrm{~S}$ state to the ground state) this transition is narrow and allows stringent tests of atomic physics. With an Einstein coefficient of $10^{-7} \mathrm{~s}^{-1}$ the transition is 14 orders of magnitude weaker than the $2^{3} \mathrm{~S} \rightarrow 2^{3} \mathrm{P}$ transition, which is the most accurately measured transition in helium (natural linewidth $1.6 \mathrm{MHz}$ ). In this contribution we first show our results, published in Science [1] and summarized already in an earlier conference proceedings [2], and then compare our isotope shift measurement with a very recent measurement of the isotope shift in the $2^{3} \mathrm{~S} \rightarrow 2{ }^{3} \mathrm{P}$ transition. We conclude with an outlook on future measurements that should allow a more accurate measurement of the isotope shift. That will then be very relevant to compare with nuclear charge radius measurements, planned on muonic ${ }^{4} \mathrm{He}^{+}$and ${ }^{3} \mathrm{He}^{+}$ and aimed to help solving the proton size puzzle.

This is an Open Access article distributed under the terms of the Creative Commons Attribution License 2.0, which permits unrestricted use, distribution, and reproduction in any medium, provided the original work is properly cited. 

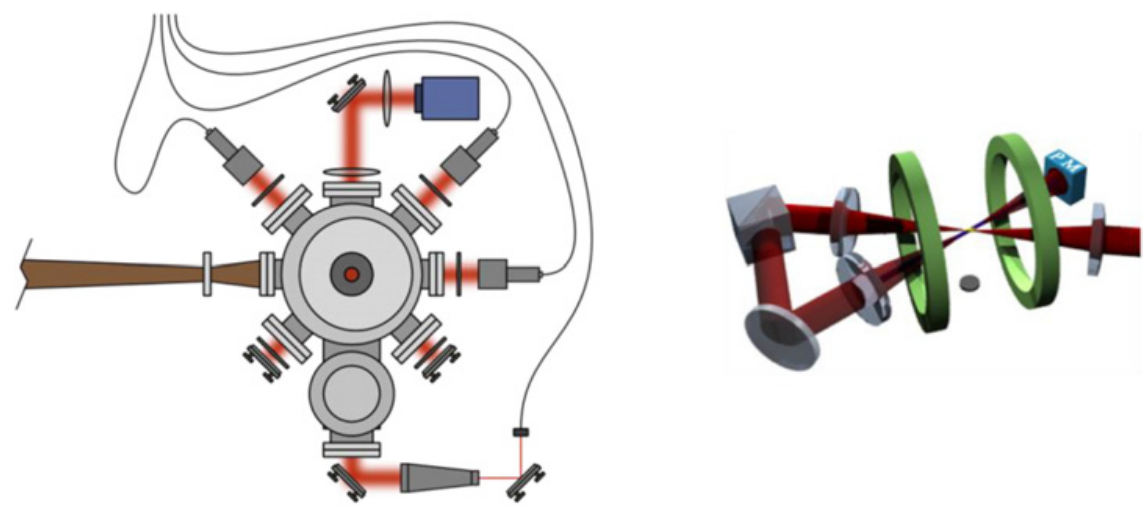

Figure 1. Experimental setup showing, left, schematically a side view of the beam machine with the end of the Zeeman slower and the UHV vacuum chamber with MOT and absorption imaging beams (all coupled by fibers), and, on the right hand side, the crossed dipole trap (at $\sim 20$ degrees) around the axial coils for magnetic trapping. A microchannel plate detector for metastable atoms is positioned underneath the trap.

$$
\text { singlet }
$$

(para-helium)

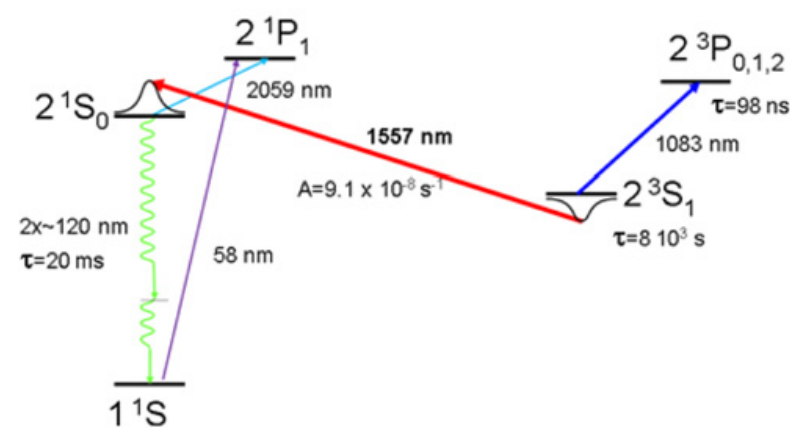

\section{triplet}

(ortho-helium)

(1)

Figure 2. Relevant energy levels, transition wavelengths, and state lifetimes in helium-4. The very weak 1557-nm magnetic-dipole transition between the $2{ }^{1} \mathrm{~S}$ and $2{ }^{3} \mathrm{~S}$ states is measured. A focused $1557-\mathrm{nm}$ laser (slightly detuned from resonance) also constitutes a trap for ultracold atoms in the $2^{3} \mathrm{~S}$ state while atoms in the $2{ }^{1} \mathrm{~S}$ state are antitrapped in 1557-nm light.Laser cooling is performed at $1083 \mathrm{~nm}$.

\section{EXPERIMENT}

Quantum degenerate gases of metastable helium are produced in the setup schematically shown in Fig. 1. Typically $3 \times 10^{8}{ }^{4} \mathrm{He}^{*}$ atoms, excited in a DC discharge to the metastable $2{ }^{3} \mathrm{~S}$ state and slowed in a Zeeman slower, are cooled and trapped in a magneto-optical to a temperature of $1 \mathrm{mK}$. Cooling and magneto optical trapping are performed at $1083 \mathrm{~nm}$ (see the level scheme in Fig. 2). Subsequently the atoms are transferred and trapped in a cloverleaf magnetic trap followed by evaporative cooling to quantum degeneracy. A ramp of $15 \mathrm{~s}$ produces condensates with more than $10^{6}$ atoms at a temperature of $1 \mu \mathrm{K}$. The fermionic helium isotope ${ }^{3} \mathrm{He}$ can also be cooled into the quantum degenerate regime (also around $1 \mu \mathrm{K}$ ) applying sympathetic cooling of ${ }^{3} \mathrm{He}^{*}$ by collisions with ${ }^{4} \mathrm{He}^{*}$ atoms. The atoms are subsequently loaded into a 1557-nm crossed dipole trap (red focused beams in the right hand part of Fig. 1). The crossed-beam dipole trap is realized by focusing both the incident and returning trap beam from a fiber laser at $1557 \mathrm{~nm}$ (far detuned from the atomic resonance) at the centre of the magnetic trap, confining the atoms at the intersection. The same laser that excites the transition is also used to 


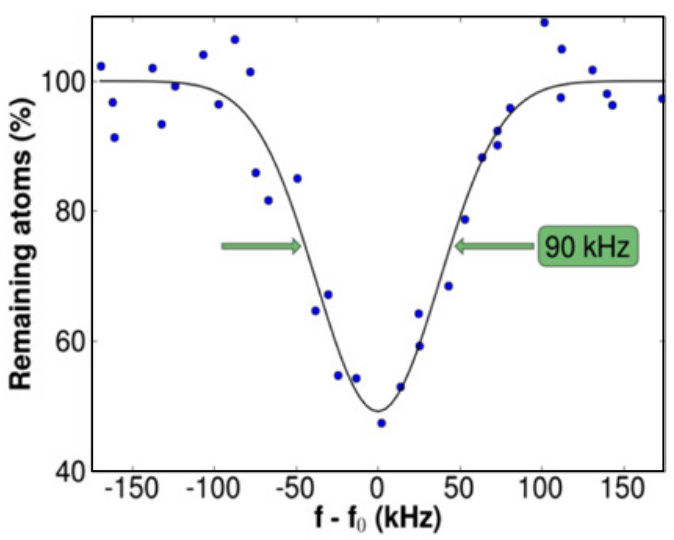

Figure 3. The observed $2{ }^{3} \mathrm{~S} \rightarrow 2{ }^{1} \mathrm{~S}$ transition for ${ }^{4} \mathrm{He}^{*}$, measured by extracting the remaining number of trapped atoms after interaction with the 1557 -nm laser for several seconds.

trap the triplet-state atoms. To allow excitation of the $2{ }^{1} \mathrm{~S}$ state, part of the $1557-\mathrm{nm}$ laser beam is frequency shifted by an acousto-optic modulator (AOM) and overlapped with the returning trap beam. A third (small) fraction of the 1557-nm laser is split off from the laser output to allow wavelength measurement by a frequency comb laser. A microchannel plate detector is positioned underneath the trap (see right hand side of Fig. 1) to provide sensitive detection of the atoms, determine the number of Bose-condensed and to measure the temperature. Absorption imaging is a second way to observe the atoms, allowing state-selective (Stern-Gerlach) and isotope-selective detection (see left hand side of Fig. 1).

Fortuitously the triplet-state atoms feel a strong attractive trapping potential at $1557 \mathrm{~nm}$, allowing trapping with $\sim 100 \mathrm{~mW}$ laser power for temperatures around $1 \mu \mathrm{K}$. The singlet-state atoms, however, feel a repulsive potential and are expelled from the trap. To measure the transition frequency, the number of trapped atoms, remaining in the trap after a few seconds of interaction with the spectroscopy beam, is measured on the microchannel plate detector. A commercial frequency comb (based on a mode-locked Er-doped fiber laser), locked to a GPS-controlled rubidium clock, provides laser-frequency calibration and measurement as well as stabilization.

\section{RESULTS}

We observed a 90-kHz wide resonance (Fig. 3). The natural linewidth is only $8 \mathrm{~Hz}$ so the observed width is due to technical imperfections. One reason for the observed width is the $75-\mathrm{kHz}$ laser linewidth during the few seconds of measurement time. Another reason is the $\sim 10 \mathrm{kHz}$ Doppler width at $1 \mu \mathrm{K}$, which in particular affects the ${ }^{3} \mathrm{He}$ fermions that, at our density and temperature, fill the trap up to the Fermi energy. The fitted transition frequencies for both isotopes, determined with $8 \times 10^{-12}$ precision $(1.5 \mathrm{kHz}$ for ${ }^{3} \mathrm{He}$ and $1.8 \mathrm{kHz}$ for ${ }^{4} \mathrm{He}$ ), agree with QED calculations but are a factor of 1000 more accurate. From the isotope shift, measured with $2.3 \mathrm{kHz}$ accuracy, we deduce a competitive value for rms charge radius of the ${ }^{3} \mathrm{He}$ nucleus (the helion) of 1.961(4) fm. This accuracy is limited by the accuracy to which the radius of the $\alpha$-particle is presently known. These results were published in 2011 [1].

\subsection{Comparison with recent results on the $2{ }^{3} \mathrm{~S} \rightarrow 2{ }^{3} \mathrm{P}$ transition}

Actually differences in squared nuclear charge radii between ${ }^{3} \mathrm{He}$ and ${ }^{4} \mathrm{He}\left(\delta \mathrm{r}^{2}\right)$ are deduced from these isotope shift measurements. For our transition this difference was $1.019(11) \mathrm{fm}^{2}$ [1]. An independent determination of $\delta \mathrm{r}^{2}$ was performed in 1995 [3]. Using the $2{ }^{3} \mathrm{~S} \rightarrow 2{ }^{3} \mathrm{P}$ transition the sensitivity to the 
Table 1. Comparison of differences in the squared nuclear charge radii between ${ }^{3} \mathrm{He}$ and ${ }^{4} \mathrm{He}\left(\delta \mathrm{r}^{2}\right)$.

\begin{tabular}{|c|c|c|}
\hline & $\delta \mathrm{r}^{2}\left(\mathrm{fm}^{2}\right)$ & Reference \\
\hline $\begin{array}{c}2{ }^{3} \mathrm{~S}-2{ }^{1} \mathrm{~S} \text { (vanRooij 2011) } \\
\text { including 2012 theory }\end{array}$ & $1.028(11)$ & {$[1,4]$} \\
\hline Nuclear few-body theory & $1.16 \pm 0.12$ & {$[1]$} \\
\hline Electron scattering experiments & $1.01 \pm 0.13$ & {$[1]$} \\
\hline $\begin{array}{c}2{ }^{3} \mathrm{~S}-2{ }^{3} \mathrm{P}_{0} \text { (Shiner 1995) } \\
\text { including 2012 theory }\end{array}$ & $1.066(4)$ & {$[3,5]$} \\
\hline $\begin{array}{c}{ }^{3} \mathrm{~S}-2{ }^{3} \mathrm{P}_{0,1,2}(\text { Cancio 2012) } \\
\text { including 2012 theory }\end{array}$ & $1.074(3)$ & {$[4]$} \\
\hline
\end{tabular}

nuclear charge radius difference is larger and a value $\delta \mathrm{r}^{2}=1.059(3) \mathrm{fm}^{2}$ was deduced from the small frequency difference between the $2{ }^{3} \mathrm{~S}_{1} \rightarrow 2{ }^{3} \mathrm{P}_{2}$ transition in ${ }^{4} \mathrm{He}$ and the $2{ }^{3} \mathrm{~S}_{1} \mathrm{~F}=3 / 2 \rightarrow 2{ }^{3} \mathrm{P}_{0} F=$ $1 / 2$ transition in ${ }^{3} \mathrm{He}$. This $3 \sigma$ difference did not worry us at the time. However, after publication of our paper new results on measurements of the complete hyperfine structure in the ${ }^{3} \mathrm{~S} \rightarrow 2{ }^{3} \mathrm{P}$ transition of ${ }^{3} \mathrm{He}$ were published [4]. Also a new and much improved quantum electrodynamics calculation of the hyperfine structure in the $2^{3} \mathrm{~S} \rightarrow 2{ }^{3} \mathrm{P}$ transition as well as the $2{ }^{3} \mathrm{~S} \rightarrow 2{ }^{1} \mathrm{~S}$ transition of ${ }^{3} \mathrm{He}$ was performed [5]. These results shifted our result a little and the new $2^{3} \mathrm{~S} \rightarrow 2{ }^{3} \mathrm{P}$ measurements confirmed the Shiner results from 1995. The present status for both transitions is shown in Table 1. A disturbing $4 \sigma$ disagreement between both transitions is present. We have no explanation for this difference.

\section{FUTURE EXPERIMENTS}

The large discrepancy between both measurements of the difference in squared nuclear charge radius between the $\alpha$-particle and the helion needs to be resolved. Moreover, there is also considerable interest in this charge radius difference from the recently observed discrepancy between proton size measurements from atomic hydrogen and muonic hydrogen spectroscopy [6]. The so-called proton radius puzzle, i.e. the observation of a proton radius that is $4 \%$ smaller than expected (5 standard deviations away from the internationally accepted CODATA value) can only be understood shifting the Rydberg constant far outside its accepted error bar or accepting that present-day QED calculations in atomic hydrogen and/or muonic hydrogen atoms are incomplete. To shed light in the proton size puzzle the CREMA collaboration at PSI, Villigen, Switzerland, will measure the $\mu^{4} \mathrm{He}^{+}$and $\mu \mathrm{g} \mathrm{He} e^{+} \mathrm{rms}$ nuclear charge radii with an accuracy of 0.5 am [7]. The measurements are planned for 2013 . These experiments are expected to improve the absolute accuracy in the rms charge radius of the $\alpha$-particle and the helion by a factor of 10 . However, the analysis will rely on QED theory for muonic helium. These measurements can tell, depending on the outcome, whether the solution of the proton size puzzle has to be found in the fact that the measurements are performed in muonic atoms rather than electronic atoms, or that the Rydberg constant was wrong $\left(\mathrm{He}^{+}\right.$spectra are less dependent on the Rydberg constant than $\mathrm{H}$ spectra) or that $\mathrm{H}$-like bound state QED is wrong. It will be very interesting to confront these results with measurements in 'normal' electronic matter such as electron scattering measurements on $\alpha$ - and helion particles and spectroscopy of $\mathrm{He}$ and/or $\mathrm{He}^{+}$.

We plan to improve on our 2011 result and measure the difference in the rms charge radius of the $\alpha$-particle and the helion with an accuracy better than $0.5 \mathrm{am}$. This accuracy will then be at the same level as anticipated in the new CREMA experiment at the muon accelerator facility at PSI, however not obtained with $\mu^{4} \mathrm{He}^{+}$and $\mu \mathrm{g} \mathrm{He}{ }^{+}$ions but with 'normal' ${ }^{4} \mathrm{He}$ and ${ }^{3} \mathrm{He}$ atoms, applying QED for normal electronic matter. To reach the projected accuracy we will need a 25 -fold improvement in the measurement of the isotope shift in the $2^{3} \mathrm{~S}_{1} \rightarrow 2{ }^{1} \mathrm{~S}_{0}$ transition at $1557 \mathrm{~nm}$. This may then also shed light on the discrepancy with the $2^{3} \mathrm{~S} \rightarrow 2{ }^{3} \mathrm{P}$ results. Our present experiment allowed an accuracy of 
(a)

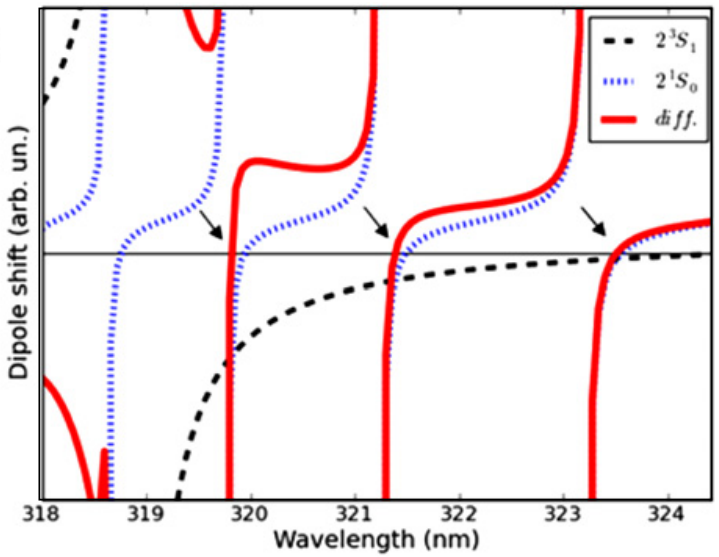

(b)

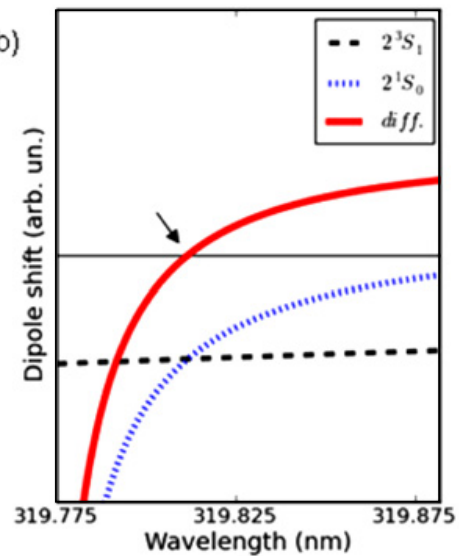

Figure 4. (a) Dipole shift (polarizability) for the $2{ }^{3} \mathrm{~S}$ state (black stripes) and the $2{ }^{1} \mathrm{~S}$ state (blue dotted) and their difference (red line), calculated from atomic energy level positions and dipole matrix elements for He. At $318.867 \mathrm{~nm}$ the $2{ }^{3} \mathrm{~S} \rightarrow 4{ }^{3} \mathrm{P}$ resonance causes the dipole shift to go to infinity for the triplets. The singlet curve shows resonances for $2{ }^{1} \mathrm{~S} \rightarrow \mathrm{n}{ }^{1} \mathrm{P}$ transitions with $\mathrm{n}=13(318.622 \mathrm{~nm}), 12(319.767 \mathrm{~nm}), 11(321.250 \mathrm{~nm})$ and 10 $(323.220 \mathrm{~nm})$. The red curve goes through zero (equal shifts for both metastable states) at the magic wavelengths $319.812 \mathrm{~nm}, 321.386 \mathrm{~nm}$ and $323.510 \mathrm{~nm}$ (see arrows). (b) enlarged part around the first magic wavelength, where the polarizability is largest and trapping requires minimum laser power.

$2.3 \mathrm{kHz}$ in the isotope shift and we aim to improve this accuracy to $0.1 \mathrm{kHz}$, still much larger than the $8 \mathrm{~Hz}$ natural linewidth. The accuracy in [1] was limited by several experimental imperfections that contributed with comparable $1 \mathrm{kHz}$ uncertainty to the total uncertainty. The most challenging innovation will be to use a separate laser for trapping the atoms. The spectroscopy laser can still be the 1557-nm fiber laser. By carefully choosing the wavelength of the trapping laser such that the AC Stark shifts (as a result of the high laser power) for the upper and lower state of the transition are equal (magic wavelength) we expect to cancel the AC Stark shift due to the trapping laser, which is the major error source. In a preliminary study (see Fig. 4) we have found 3 magic wavelengths at which atoms are trapped and where the polarizability is large enough. The most promising wavelength is $319.812 \mathrm{~nm}$ where trapping will require $0.5 \mathrm{~W}$ of laser power. Although this magic wavelength is only $1 \mathrm{~nm}$ detuned from the $2^{3} \mathrm{~S} \rightarrow 4^{3} \mathrm{P}$ transition, we calculated that the lifetime of the atoms in the trap will be several seconds, long enough to perform the experiment. It is actually an important advantage of helium that these experimental numbers can be calculated ab-initio. To exploit the reduced AC Stark shift fully, we plan to narrow down the $1557-\mathrm{nm}$ laser linewidth, from its present $75 \mathrm{kHz}$ value to $\sim 5 \mathrm{kHz}$, the short-time linewidth of our spectroscopy laser. This will increase the excitation rate, allowing shorter measurement time and improved statistics.

\section{References}

[1] R. van Rooij, J.S. Borbely, J. Simonet, M.D. Hoogerland, K.S.E. Eikema, R.A. Roozendaal, W. Vassen, Science 333, 196 (2011).

[2] W. Vassen, in: Proceedings of the 20th International conference in laser spectroscopy (ICOLS 2011), eds. W. Ertmer, R. Scholz (Logos Verlag Berlin GmbH, 2011).

[3] D. Shiner, R. Dixson and V. Vedantham, Phys. Rev. Lett. 74, 3553 (1995).

[4] P. Cancio Pastor, L. Consolino, G. Giusfredi, P. De Natale, M. Inguscio, V.A. Yerokhin, K. Pachucki, Phys. Rev. Lett. 108, 143001 (2012).

[5] K. Pachucki, V.A. Yerokhin, P. Cancio Pastor, Phys. Rev. A 85, 042517 (2012).

[6] R. Pohl et al., Nature 466, 213 (2010).

[7] A. Antognini et al., Can J. Phys. 89, 47 (2011). 